\title{
Phaeochromocytoma and catecholamine induced cardiomyopathy presenting as heart failure
}

Suhrud H Sardesai, Anthony J Mourant, Yasothai Sivathandon, Richard Farrow, David O Gibbons

\begin{abstract}
Phaeochromocytoma is rare and usually presents as paroxysmal or sustained hypertension; none the less, it can also cause severe acute pulmonary oedema in normotensive individuals.

Six patients with phaeochromocytoma presenting in Cornwall and West Devon between 1982 and 1986 are described. Five of them died of pulmonary oedema within 24 hours of the onset of symptoms. At necropsy all five had normal sized hearts and in the four hearts examined by histology there was evidence of catecholamine induced heart disease in the form of focal myocardial necrosis. The sixth patient presented with arterial spasms and pulmonary oedema. Surgical removal of the causative tumour was successful in this patient.
\end{abstract}

The commonest manifestations of phaeochromocytoma are paroxysmal or sustained hypertension with paroxysms of adrenergic stimulation, causing palpitation, headaches, sweating, pallor, tremors, and anxiety. Phaeochromocytomas account for about $0.04 \%$ of cases of hypertension, ${ }^{2}$ but there is a distinct group of patients with phaeochromocytoma who remain normotensive despite active metabolites being secreted. ${ }^{34}$ Sometimes high blood pressure may fall back to normal values because of myocardial damage, ${ }^{5}$ and normotensive patients who do not have paroxysmal symptoms may be more likely to die as a result of adrenergic heart disease because the diagnosis is not suspected. ${ }^{6}$ Although rare, hypotension can occur when $\beta$ adrenergic stimulation overrides $\alpha$ adrenergic stimulation. $^{3}$

Acute pulmonary oedema is less widely recognised as a feature of phaeochromocytoma. We report six such patients, of whom five presented with acute pulmonary oedema.

Royal Cornwall Truro, Cornwall

$S$ H Sardesai

A J Mourant

Y Sivathandon

R Farrow

West Cornwall Hospital, Penzance, Cornwall D O Gibbons

Correspondence to

Dr Anthony J Mourant,

Dr Anthony J Mourant,

Royal Cornwall Hospital

TR1 3LJ.

Accepted for publication

19 January 1990

( min), pyrexial (axillary tempera $40^{\circ} \mathrm{C}$ ), and cyanosed. She was normotensive (blood pressure $130 / 80 \mathrm{~mm} \mathrm{Hg}$ ) and the jugular venous pressure was not raised. Heart sounds were unremarkable and there were bilateral scattered crepitations. The chest $x$ ray showed a slightly enlarged cardiac shadow and pulmonary oedema. The electrocardiogram showed sinus tachycardia, poor $\mathbf{R}$ wave progression in the chest leads, and non-specific $T$ wave changes. Full blood count and electrolytes were normal and analysis of arterial blood gases showed $\mathrm{Po}_{2} 9 \cdot 8 \mathrm{kPa}, \mathrm{PCO}_{2} 3 \cdot 2 \mathrm{kPa}$, and $\mathrm{pH}$ $7 \cdot 2$. She deteriorated rapidly and despite resuscitative measures she died within two hours of admission.

At necropsy the heart weighed $285 \mathrm{~g}$ (normal $250-400 \mathrm{~g}$ ) with minimal dilatation of both ventricles. No significant lesions were found in the coronary arteries and histological examination of the heart showed patchy muscle fibre necrosis with phagocytosis. Severe haemorrhagic oedema was found in both lungs, which showed no evidence of acute pulmonary infarction. A tumour $4 \mathrm{~cm}$ in diameter was found in the right adrenal gland and histology showed this to be a phaeochromocytoma.

\section{PATIENT 2}

A 63 year old woman was admitted with a history of chest pain and breathlessness for two hours. The week before she had presented to the local cottage hospital with malaise, pedal oedema, and non-specific chest discomfort. She had had no other significant problems in the past and had never had hypertension. On admission she was pale with sinus tachycardia of 146 beats/min and blood pressure of $110 / 70$ $\mathrm{mm} \mathrm{Hg}$. Jugular venous pressure was $3 \mathrm{~cm}$ above the sternal angle and auscultation showed a gallop rhythm. She had widespread crepitations in both lung fields. The electrocardiogram showed no evidence of myocardial infarction or left ventricular hypertrophy and the chest $x$ ray was consistent with pulmonary oedema. She was treated with frusemide, amoxycillin, and dobutamine but she did not respond to these measures and died within eight hours of admission.

At necropsy the heart weighed $350 \mathrm{~g}$ (normal $250-400 \mathrm{~g}$ ) and was macroscopically normal. Histological examination of the myocardium showed vacuolar and focal necrosis with an inflammatory reaction. The coronary arteries were normal. The airways contained frothy liquid and the lungs were moist on section with microscopic changes consistent with acute pulmonary oedema. The right adrenal gland was replaced by a phaeochromocytoma weighing $70 \mathrm{~g}$ with typical histology. 
rheumatoid arthritis started getting "funny turns" in 1976 when she was 29. Episodes lasted between two and 30 minutes when she experienced palpitation, chest tightness, and breathlessness. She was extensively investigated and initially it was felt that her symptoms were caused by refractory supraventricular tachycardia. Subsequent electrophysiological studies showed an atrioventricular accessory pathway, although later it was thought that this could not explain her various symptoms and the different types of ventricular and supraventricular tachycardias seen on Holter monitoring. Urinary concentrations of 4-hydroxy3-methoxymandelic acid were assayed on numerous occasions but were always normal. Provocation tests with glucagon and tyramine were also within normal limits.

Symptoms settled spontaneously but in May 1986 she was admitted to hospital unconscious, tachycardic, and tachypnoeic after being resuscitated after a sudden collapse at home. She was found to have gross pulmonary oedema and was deeply unconscious with evidence of anoxic brain damage. She died shortly after admission despite full supportive treatment.

At necropsy the heart size was normal ( $300 \mathrm{~g}$ ) and the coronary arteries were widely patent. The air passages contained a moderate excess of frothy mucus. The lungs were collapsed with considerable frothy oedema and congestion. Histological examination of the heart showed multiple foci of myocardial cell necrosis and surrounding infiltration with acute inflammatory cells. A phaeochromocytoma weighing $200 \mathrm{~g}$ and $7 \mathrm{~cm}$ in diameter was found in the right adrenal gland.

\section{PATIENT 4}

A 44 year old woman who was 36 weeks' pregnant was admitted in October 1986 with hydramnios and an abnormal glucose tolerance test. Her first two deliveries had been normal and although the third had been uncomplicated the baby had spina bifida and died soon after birth. Subsequently she had three miscarriages (fetal ages between eight and nine weeks). In 1985 she was investigated for episodic palpitation which she had had for 20 years. Supraventricular tachycardia was documented and no cause was sought.

Labour was induced by artifical rupture of membranes because of the hydramnios but failed to progress. At subsequent caesarean section a live baby was delivered but postoperatively tachycardia, hypotension, and severe pulmonary oedema developed in the mother. She had a cardiac arrest but was successfully revived and transferred to the intensive care unit. Despite full cardiopulmonary support, she had a further cardiac arrest and died within 12 hours of the caesarean section.

At necropsy the heart size was normal (295 g). The valves and coronary arteries were completely normal. Myocardial histology showed focal necrosis and inflammatory cells. The airways and lungs showed changes consistent with pulmonary oedema. The right adrenal gland was replaced with a dark brown tumour weighing $125 \mathrm{~g}$ and histology showed this to be a phaeochromocytoma with haemorrhagic and necrotic areas.

\section{PATIENT 5}

A 69 year old retired quarry worker collapsed and died while working on his farm. A short time before he had complained to his family of feeling generally unwell and breathless. There was no relevant past medical history and the general practitioner's records showed him to be normotensive.

At necropsy the heart size was normal $(375 \mathrm{~g})$. The coronary arteries showed a few patches of atheroma and the aorta showed moderate atherosclerosis. Microscopic examination of the heart was not performed. Both lungs were oedematous. A tumour $7 \mathrm{~cm}$ in diameter was found attached to the posterior abdominal wall, near the bifurcation of the aorta. Histologically this was found to be a phaeochromocytoma.

\section{PATIENT 6}

This 48 year old crane driver was admitted to hospital in 1981 with sudden onset of gangrene of the left forefoot, headaches, and breathlessness. He was normotensive and had normal heart sounds. The chest $x$ ray showed slight cardiomegaly and pulmonary oedema, which responded to conventional diuretic treatment. An attempted embolectomy via the left popliteal artery produced no clot and the possibility of a vasospastic phenomenon was considered. A few days later a left below knee amputation was performed and histological examination of this showed no evidence of arterial disease. After the operation he was referred to the physicians and had several further investigations including an electrocardiogram, echocardiogram, and measurement of urinary concentrations of 4-hydroxy3-methoxymendelic acid-all were within normal limits.

One year after the initial presentation he had a left lumbar sympathectomy performed because of persistent problems with a cold blue stump.

A second exploration was attempted two years after the initial presentation because of continuing pain in the stump, with a view to excision of the long saphenous nerve. During the operation pulmonary oedema developed. Postoperatively his blood pressure was $110 / 70$ $\mathrm{mm} \mathrm{Hg}$ and the urinary concentration of metadrenaline was raised $(8.2 \mu \mathrm{mol} / 1$ (normal $<5 \mu \mathrm{mol} / \mathrm{l})$ ). A computed tomogram showed a right adrenal tumour. At operation a phaeochromocytoma measuring $4 \times 5 \mathrm{~cm}$ was removed. He made a good postoperative recovery.

\section{Discussion}

Cardiovascular injury induced by sympathomimetic amines has been well recognised for some time in both humans and experimental animals. ${ }^{78}$ It is also recognised that myocardial lesions produced by high doses of catecholamines are similar in all species including humans. ${ }^{9}$ The finding of foci of myocardial 
cellular necrosis is consistent and predominant. Initial lesions show diffuse oedema and minor alteration of myocardial nuclei. Later, focal myofibril degeneration with loss of striation of individual myocytes is seen. ${ }^{10}$ Inflammatory cellular infiltration takes place in response to this. When phaeochromocytomas were transplanted into rats the heart consistently showed multifocal lesions of enhanced interstitial and replacement fibrosis, granularity of the cytoplasm and contraction band necrosis, and mixed inflammatory infiltrates six weeks after transplantation. ${ }^{11}$ More advanced lesions had more fibrosis and did not show inflammatory cells. $^{10}$

During the earlier stages, the myocardium in patients with phaeochromocytomas resembles that seen in patients treated with noradrenaline (norepinephrine) and the term "norepinephrine myocarditis" has been used to describe the cardiac lesion..$^{72}$ Others have used the same term because of functional improvement in the cardiac condition after surgical removal of the phaeochromocytoma. ${ }^{13}$ It is perhaps more appropriate to use only the term "focal myocardial necrosis" because the inflammation is always a secondary process ${ }^{14}$ or "catecholamine induced cardiomyopathy" which is the term that we have used. Previously it has been suggested that the incidence of clinically significant catecholamine induced cardiomyopathy is far less than that seen at necropsy..$^{15}$

Studies of catecholamine induced cardiomyopathy have shown a global reduction in myocardial pump function caused by a combination of down-regulated $\beta$ receptors and a net reduction in viable myofibrils. ${ }^{16}$ Rabbit hearts with catecholamine induced cardiomyopathy had a reduced inotropic sensitivity to noradrenaline and also a reduced response to calcium chloride infusion. The pathogenesis of catecholamine induced cardiomyopathies is probably multifactorial. Catecholamine induced vasospasm leading to hypoxia is implicated and supported by the finding that more severe lesions are located near the cardiac apex-an area of peripheral coronary circulation. ${ }^{17}$ Excess noradrenaline also induces changes in permeability of the sarcolemmal membrane leading to increased calcium influx. This excess intracellular calcium has a direct toxic action giving rise to cellular necrosis. Although calcium is undoubtedly important, it is not the exclusive factor causing damage. ${ }^{1819}$ There is evidence that the oxidised products of catecholamines may be toxic and contribute to the damage. ${ }^{19}$ It has also been proposed that the injury process might involve damage by free radicals. ${ }^{20}$

The commonest manifestations of phaeochromocytoma are of paroxysmal or sustained hypertension or symptoms of paroxysmal adrenergic stimulation, such as palpitation, headaches, anxiety, sweating, and tremors; rarer presentations, such as acute abdomen, cerebrovascular events, myocardial infarction, hyperglycaemia, postural hypotension, shock, and heart failure have been reported.

We described five normotensive patients with phaeochromocytoma presenting with pul- monary oedema as a dominant feature. In the patient who died suddenly pulmonary oedema was seen at necropsy. None had confirmed hypertension. Five of the patients had normal sized hearts. Histological examination undertaken in four of our patients showed extensive focal myocardial necrosis.

The diagnosis of phaeochromocytoma was considered in two of the six patients described. One gave a history of paroxysmal symptoms before presenting with severe heart failure as a terminal event (patient 3); the other had additional vascular spasms together with pulmonary oedema (patient 6). Both of these patients had normal urinary concentrations of 4-hydroxy-3-methoxymandelic acid initially but neither was examined by computed tomography when they first presented and this investigation might have led to the correct diagnosis being reached earlier. In patient 6 , a subsequent computed tomogram confirmed the presence of an adrenal phaeochromocytoma. We now use computed tomography in the initial assessment of patients in whom the diagnosis of phaeochromocytoma is strongly suspected despite normal urinary concentrations of 4-hydroxy-3-methoxymandelic acid. The remaining four patients presented rapidly and died before relevant investigations could be performed.

Our experience indicates that phaeochromocytomas cause clinically important catecholamine induced cardiomyopathy more commonly that has been thought. In patients with phaeochromocytoma presenting with acute heart failure the prognosis can be very poor because of the extensive focal myocardial damage.

In patients presenting with heart failure without any obvious cause, the diagnosis of phaeochromocytoma should be considered. Urinary concentrations of 4-hydroxy-3methoxymandelic acid should be measured and computed tomography should be undertaken as soon as possible.

We thank the following consultants for the inclusion in this report of patients under their care: Dr A J Marshall, Dr E W Hughes, Dr B J Prout, and Dr J L Dunscombe.

1 Ross EJ, Griffith DNW. The clinical presentation of pheochromocytoma. $Q J$ Med 1989;266:485-96.

2 Kuska J, Kokot F, Drab M, Drobisz M. Frequency of incidence of particular etiological forms of arterial hyperincidence of particular etiological forms of arterial hyper-
tension based on 5155 cases analysis. Kardiol Pol 1988; tension basec

3 Page LB, Raker JW, Berberich FR. Pheochromocytoma with predominant epinephrine secretion. Am J Med 1969; 47:648-52.

4 Garcia R, Jennings JM. Pheochromocytoma masquerading as a cardiomyopathy. Am J Cardiol 1972;29:568-71.

5 Baker G, Zeller NH, Weitzner S, Leach JK, Albuquerque NM. Pheochromocytoma without hypertension present ing as cardiomyopathy. Am Heart $J$ 1972;83:688-93.

6 Moorhead EL, Caldwell JR, Kelly AR, Morales AR. The diagnosis of pheochromocytoma. JAMA 1966;196: 1107-13.

7 Szakacs JE, Cannon A. 1-Norepinephrine myocarditis. Am J Clin Pathol 1958;30:425-34

8 Handforth CP. Isoproterenol-induced myocardial infarction in animals. Arch Pathol 1962;73:83-7.

9 Haft JI. Cardiovascular injury induced by sympathetic catecholamines. Prog Cardiovasc Dis 1974;17:73-86.

10 Van Vliet PD, Burchell HB, Titus JL. Focal myocarditis associated with pheochromocytoma. $N$ Engl J Med 1966; 274:1102-8.

11 Rosenbaum JS, Billingham ME, Ginsberg R, et al. Cardiomyopathy in a rat model of pheochromocytoma Morphological and functional alterations. $\mathrm{Am}$ j Cardiovasc Pathol 1988;1:389-99. 
12 Kline IK. Myocardial alterations associated with pheochromocytomas. Am J Pathol 1961;38:539-51.

13 Imperato-McGinley J, Gautier T, Ehlers K, Zullo MA Goldstein DS, Vaughan ED. Reversibility of catecholamine induced dilated cardiomyopathy in a child with a pheochromocytoma. $N$ Engl J Med 1987;316:793-7.

14 Bloom S. Catecholamine cardiomyopathy [Letter]. $N$ Engl J Med 1987;317:900.

15 Yankopoulos NA, Montero AC, Curd WG, Kahil ME, Condon RE. Observations on myocardial function during chronic catecholamine oversecretion. Chest 1974;66: 585-7.

16 Fripp RR, Lee JC, Downing SE. Inotropic responsiveness of the heart in catecholamine cardiomyopathy. Am Heart $J$ 1981;101:17-21.

17 Takanori C, Tanimura A, Saito Y. Catecholamine-induced cardiomyopathy accompanied with pheochromocytoma. Acta Pathol Jpn 1987;37:123-32.

18 Bloom S, Davis DL. Calcium as mediator of isoproterenolinduced myocardial necrosis. Am J Pathol 1972;69: 459-70.

19 Rona G. Catecholamine cardiotoxicity. J Mol Cell Cardiol 1985; 17:291-306.

20 Singal PK, Kapur N, Dhilon KS, et al. Role of free radicals in catecholamine induced cardiomyopathy. Can J Physiol Pharmacol 1982;60:1390-7.

\section{VIEWS FROM THE PAST William Evans}

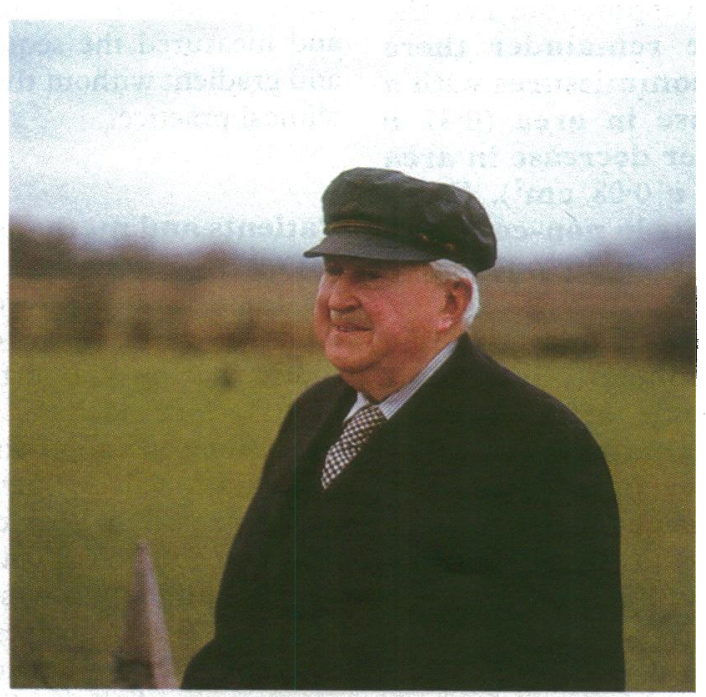

William Evans's obituary was printed in the British Heart Journal of January 1989. Here he is photographed by me in 1975 on his farm in Tregaron, Cardiganshire, where he was born in 1895 and died in September 1988, aged ninety two. Wallace Brigden, writing in Munk's Roll, says that "On retirement from the London Hospital he returned to the Wales he loved. The Principality reciprocated in making him High Sheriff of Cardigan and an Honorary Druid. He placed both of these honours above the legion of professional ones which came his way. He was not the most eminent cardiologist of his time but one of the most unusual, well known and well liked. In retrospect, medicine in general and cardiology in particular seemed to be only an interlude, albeit an absorbing and exciting one which occurred between childhood in the Teify Valley overlooked by Cambrian hills and the same unchanged scene in his twenty five years of retirement." AUBREY LEATHAM 